\title{
Preparing regenerative therapies for clinical application: proposals for responsible translation
}

\author{
Shane A Shapiro*,1,2, Cambray G Smith ${ }^{3}$, Jennifer R Arthurs ${ }^{2}$ \& Zubin Master²,3 \\ ${ }^{1}$ Department of Orthopedic Surgery, Mayo Clinic, 4500 San Pablo Road, Jacksonville, FL 32224, USA \\ ${ }^{2}$ Mayo Clinic Center for Regenerative Medicine, 200 First Street, SW, Rochester, MN 55905, USA \\ ${ }^{3}$ Biomedical Ethics Research Program, Mayo Clinic, 200 First Street, SW, Rochester, MN 55905, USA \\ *Author for correspondence: shapiro.shane@mayo.edu
}

\section{'We offer a model founded on multispecialty expertise that can serve future caregivers employing validated cell therapies in multiple healthcare delivery environments."}

First draft submitted: 10 December 2018; Accepted for publication: 4 January 2018; Published online: 17 January 2019

Keywords: bone marrow aspiration and concentration $\bullet$ multidisciplinary expertise $\bullet$ patient education • PRP • regenerative medicine $\bullet$ registries $\bullet$ regulatory $\bullet$ stem cell

Regenerative therapies have exploded in popularity due primarily to two factors: unmet patient needs and enthusiasm about the potential of cell-based therapies to meet those needs. Despite consisting largely of both discovery and translational research, regenerative medicine is fast becoming a medical specialty in its own right, recognized for its potential to advance care for chronic conditions [1-5]. In a relatively short period of time, cellular therapies are beginning to be developed, peer reviewed and validated for a wide variety of medical conditions [6-8].

Although medicine has historically lagged behind other industries in implementing disruptive transformative therapies, US FDA Commissioner Scott Gottlieb has stated that, to achieve the goals of regenerative medicine, the FDA is "taking steps to advance an innovative framework for how we intend to apply the existing laws and regulations that govern these products. Our aim is to make sure we're being nimble and creative when it comes to fostering innovation, while taking steps to protect the safety of patients" [9]. The 21st Century Cures Act of 2016 is an effort to modernize and speed up the translation of regenerative therapies for unmet patient needs [10]. The legislation specifically charges FDA with developing expedited pathways bringing regenerative therapies to market. As the pace of regenerative discovery and innovation continues to accelerate, it will be incumbent upon the healthcare industry to prepare itself to translate and apply such therapies into patient treatments. As such, in the dawning phases of cell therapy, we anticipate a need to explore novel options to practice this new subspecialty of medicine.

In this article, we describe the implementation of a clinical program designed to translate valid regenerative therapies (cellular and noncellular) for multispecialty practice with the intent to ease traditional obstacles to delivering quality regenerative care by educating patients, implementing quality control and procedural integrity while collecting real-world evidence (RWE) to validate its use.

\section{Current state of regenerative medicine}

Regenerative medicine has increasingly been recognized as an emerging field of medicine since bone marrow transplant research has led to a greater understanding of stem cell therapy. California voters approved the creation of the California Institute of Regenerative Medicine in 2004 and funded the initiative with US $\$ 3.4$ billion in public dollars [7]. Many other states (e.g., MD, MA, CT, MN and NY) have followed suit and the biotech industry has created its own sector of companies focused on research, development and commercialization of regenerative cellular and other therapies to transform the practice of medicine [11].

The list of regenerative medicine successes is beyond the scope of this paper, but several major milestones have been achieved including increase in clinical trials, regulatory approvals leading to early commercial translation and the creation of centers of research excellence - all of which drive regenerative medicine toward clinical success [12]. 
Some notable examples include autologous culture expanded keratinocytes to treat several patients with junctional epidermolysis bullosa [13]. This cell therapy treatment has led to regeneration of the epidermis [14]. Cells have also been used to regenerate skin after burn wounds and a recent spray-on skin product using a patient's own skin cells has been given FDA approval [15]. T-cell-based immunotherapies have also been FDA approved to treat certain malignancies [16-18]. Similarly, two cell-based products have been given marketing approval by the European Medicines Agency for treatment of severe limbal stem cell deficiency of the eye and Crohn's fistulas [19,20].

Point of care, same surgical procedure cell therapies have also shown some early success and are increasingly used in the novel field of orthobiologics. Bone marrow aspiration and concentration for avascular necrosis of the femoral head in conjunction with surgical core decompression has improved patient outcomes and can prevent hip replacement in early stage cases [21]. Finally, autologous platelet therapies for androgenetic alopecia and knee pain from arthritis continue to show promise in randomized clinical trials and meta-analyses with subsequent incorporation into routine orthopedic and dermatologic practices [22,23]. It bears noting that not all novel cell therapies have been met with success as there are numerous examples of failure in this space as would be expected in any novel scientific field [24]. Consequently, standards put forth by the International Alliance for Biological Standardization are critical to ensure safety and quality [25].

Despite such successes and attempts to validate regenerative medicine interventions, there lies an industry fueled by hype and misinformation, attempting to sell invalid and unapproved stem cell and regenerative therapies, with growing markets worldwide, perhaps most notably in the USA [26-28].

\section{The Regenerative Therapeutics Program}

As regenerative medicine therapies advance and become approved, and as the practice transitions into its own medical specialty, multiple medical specialists will be needed to perform regenerative therapies requiring the training of clinicians [5]. Such practices have already evolved somewhat out of necessity. One example includes the Alpha Stem Cell Clinics which are a network created by the California Institute of Regenerative Medicine with expertise in clinical trials related to cell therapy $[6,7]$. We offer a model founded on multispecialty expertise that can serve future caregivers employing validated cell therapies in multiple healthcare delivery environments.

The Regenerative Medicine Therapeutics Program is being pioneered by the Mayo Clinic Center for Regenerative Medicine and addresses three of the traditional obstacles to delivering regenerative care, which include deficits in patient education about regenerative therapies, the challenge of studying and treating a wide variety of both common and less-common diseases with regenerative therapies [8], and the lack of high-quality RWE in regenerative medicine in support of translation.

The Regenerative Therapeutics Program is a multidisciplinary program with opportunities for all medical specialties to participate in the translation of regenerative and cell-based therapies into practice. It has three main components that attempt to address the barriers described above: the Regenerative Medicine Consult Service (RMCS), the Therapeutic Suites - a multidisciplinary surgical hybrid procedure suite for cell therapy applications, and the Regenerative Evidence-Based Outcomes Registry (REBO; see Box 1).

\section{Regenerative Medicine Consultation Service}

One of the major issues today in regenerative medicine is the increased confusion of patients navigating legitimate stem cell and regenerative medicine options from ones which lack a clear evidence base [26,29]. The concept of a consultation service (originally defined as a counseling service) was first introduced as a specialty service to communicate specialized information about stem cells and regenerative medicine to patients [30]. To circumvent misinformation about unapproved stem cell treatments, and to educate and navigate patients toward scientifically founded options, we have developed the RMCS [1-3].

The RMCS is a clinical service that allows patients seeking information regarding regenerative therapies to speak with a clinical consultant with expertise in both the patients' organ-specific condition and regenerative medicine. Such consultations can be via telephone or in person. This service began in 2011 at the Mayo Clinic's Rochester, MN campus and started in Florida in 2016. The RMCS serves two main goals: as an information source and patient navigation.

During conversations with consultants, patients are provided a medical evaluation to determine whether a regenerative medicine protocol is appropriate. Patients also receive education about regenerative medicine, including the state of stem cell research for their particular condition. This also involves correcting misinformation about the unapproved stem cell therapy marketplace. For some patients, this is an eye opening experience as they remain largely 
Box 1. Overview of the Regenerative Therapeutics Program.

Regenerative Medicine Consult Service

- Given the hyperbolic claims and misinformation surrounding cell-based therapies, our consultation service aims to circumvent misinformation about cellular therapies through education and navigates patients toward scientifically-based clinical options of care or research.

Regenerative Medicine Therapeutic Suites

- The therapeutic suites are hybrid medical and surgical suites equipped with cell harvest, application, storage and receiving capabilities.

- Based on unmet patient need, we have implemented limited cell-based therapeutic options for certain medical conditions and are attempting to offer such options while simultaneously informing patients of their likelihood of success and conducting clinical trials.

- Early applications exist in the fields of orthopedics, dermatology, plastic surgery and wound care.

Regenerative Evidence Based Outcomes Registry

- Although there is excitement to capture RWE, organizations are applying real-world data with clinical study data to further the development of regenerative medicine products or to use this information to inform patients about regenerative options.

- In addition, rather than focusing exclusively on clinical outcome data and omitting patient perceptions or their understanding of cell-based therapies, Regenerative Evidence Based Outcomes Registry focuses on incorporating both clinical outcomes and social science data.

- We intend to combine real-world data with ongoing clinical studies to advance regenerative medicine therapeutics. Data can be pooled with that of other practitioners and investigators.

unaware of the current state of research and have received inaccurate information from websites and other sources. Consultants routinely offer clinical and research referrals and educational resources to patients. Patients learn about this service from internet searches, internal Mayo Clinic referrals or referrals from external providers. If an FDA approved or FDA allowed procedure is available to the patient, it will be offered as treatment in the Therapeutic Suites. Several of the lessons learned from this service include the importance of standardizing the documentation of consult notes and ensuring the systematic provision of educational resources among consultants, in addition to improving ways to ensure patient follow-up for recommended regenerative medicine clinical services and research studies. A systematic analysis of the RMCS is underway and will be reported in a subsequent manuscript.

Yet patient education on the unapproved stem cell market remains scarce [31] and more recently, infomercials disguised as educational seminars for the public are widespread [29]. Although it remains unknown, it is expected that some patients would conflate the RMCS with educational seminars put on by clinics giving them equal footing. This, and other practices, makes it difficult for patients to distinguish credible sources of information from those whose main motives are to sell an unapproved intervention. The RMCS avoids several of the key features found among infomercials including overemphasis of medical benefits with virtually no acknowledgements of risks of clinical interventions, the hard sell of a product or service deal only offered at the seminar and misinformation about FDA approval of products [29]. These aspects of stem cell education must be relayed to patients so they can recognize and appropriately scrutinize sources of information. Care must also be given to avoid therapeutic misconception as most regenerative medicine protocols still exist only in the research space.

\section{Regenerative Medicine Therapeutic Suites}

The Therapeutic Suites are novel hybrid medical and surgical procedure suites fully equipped for regulatory compliant cell harvest, application, storage, receiving and quality compliance oversight. The primary objective is to provide a safe space for medical procedures currently utilizing autologous, point of care (same patient, same surgical procedure) cell therapies allowed under the current regulatory framework specifically pertaining to the same surgical procedure exception. The same surgical procedure exception finalized guidance states that human cells or cell and tissue products (HCT/Ps) can be utilized therapeutically in a medical procedure provided they are the patient's own HCT/Ps and no manipulation or processing of the cells or tissues beyond rinsing, cleansing, sizing and shaping are performed [32]. Several medical specialties are already applying medical procedures that make therapeutic use of such HCT/Ps and have utilized the Therapeutic Suites including orthopedic and plastic surgery, pain medicine, dermatology, physical medicine and rehabilitation, transfusionists, hematologists and oncologists. In addition to the performance of such compliant procedures, the Therapeutic Suites host seven ongoing clinical trials, as well as five FDA Investigational New Drug Applications [33,34]. 
Many compliant and minimally manipulated/homologous use therapies still lack robust evidence and thus cannot be regularly recommended as part of routine practice. Others are steadily working toward the premarket approval that FDA requires for new biological drugs while some therapies qualify only to be 'registered' with the FDA. A discussion of these differences is outside the scope of this paper. At this time, in this perspective, we offer no opinion for which treatments have been validated and only advocate for a multidisciplinary collaborative approach to regenerative medicine which must include a range of laboratory, scientific, clinical and ethics experts. We must also be mindful that while preclinical success can be high, the majority of early stage clinical trials fail with a final approval being about $10 \%[35,36]$. This is separate from postmarket safety events of various clinical products [37]. Realistic timelines of marketing regenerative products need to be conveyed accurately to patients.

Given that many of these therapies are in their early stages (although with published records of safety), patient education is imperative to ensure continued understanding of why the procedure is being performed, what outcomes they can expect and that they understand any risks associated with the therapies. Ensuring that patients understand the difference between standards-of-care compared with those protocols with a record of safety, but still as yet undetermined efficacy, will continue to be a guiding principle of the Regenerative Therapeutics Program. In addition to educating patients, outcomes and other information should be collected, analyzed and used to develop an evidence base for regenerative therapeutics.

\section{RWE \& the REBO Registry}

The 21st Century Cures Act contains two areas pertinent to regenerative medicine for the purpose of expediting FDA approval of regenerative therapies to meet unmet patient needs. The first is the Regenerative Medicine Advanced Therapy designation, which allows for expedited FDA review of designated therapies that address 'serious or life-threatening' conditions [10]. To date, more than 20 cell therapies have been granted Regenerative Medicine Advanced Therapy status by the FDA [38]. The second is the acceptance of RWE arising from real-world data [39].

The FDA defines RWD as "data relating to patient health status and/or the delivery of healthcare routinely collected from a variety of sources" such as Electronic health records, registries, and electronic devices, thus RWE as "the clinical evidence regarding the usage, and potential benefits or risks, of a medical product derived from analysis of RWD" [39]. Although double-blind, placebo, randomized control trials have been used as the gold standard of scientific evidence, they have several shortcomings. They are conducted in tightly-controlled homogenous populations whose physical (e.g., absence of comorbidities) and/or behavioral characteristics may not be representative of a heterogeneous population, and they are unable to generate the type of evidence (individual genetic, environmental and behavioral information) needed for precision (individualized) medicine [40]. Additionally, they are financially and practically difficult to conduct [40]. One way to collect RWD and generate RWE is through high-quality registries. Registry data has existed for many years in specialties which track outcomes related to multiple medical conditions [41]. Commonly, such registries will track validated patient outcomes.

In order to contribute to high-quality RWD and RWE, we have created the REBO registry to track our patients over time. Unlike traditional outcomes registries, we include additional elements that incorporate ethics and social information related to health outcomes of these innovative therapies. The registry is built on a digital platform that can be customized to the needs of the practitioner as well as the healthcare field as a whole. This includes the ability to analyze the effect of medical comorbidities as well as behavioral concerns and mental health. The registry also weaves several medical and humanities specialties while also educating and informing patients.

Health outcomes data can include standardized measures such as quantitative digital cartilage mapping with MRIs, 3D wound measurements, computerized hair density and digital hair counting software, and patient reported outcomes which exist for almost every medical specialty. Other measures including psychometrics, qualitative, cognitive and health surveys are necessary in order to measure patient outcomes. Examples of these include pain, fatigue, physical functioning, emotional distress and social role participation, all which can have a major impact on quality-of-life across a variety of chronic diseases.

Collecting ethical and societal data, especially related to patient perceptions of regenerative therapies, will be valuable for trying to understand patient health behaviors and medical decision making in the context of regenerative medicine. Example questions include: why are you interested in a stem cell treatment for your condition? How did you find out about a stem cell treatment for your condition? Have you contacted a clinic to speak to them about a stem cell treatment for your condition? Did your practitioner explain to you all of the available options for your condition including standard of care as well as alternatives to a specific cell therapy treatment? This information 
has practical application including designing better information for patients and enhancing the informed consent process.

REBO is designed not only to be a data collection repository, but also a digital learning platform that can show prospective patients outcome data in terms of effective treatments based on clinical conditions and other factors in order to help guide patient decision making. Such RWE will be helpful to other researchers looking to design clinical trials and also to regulatory authorities to accompany other clinical data when considering regulatory approval of regenerative products. Our approach is to provide RWD to complement the scientific evidence generated from traditional clinical trials when developing regenerative medicine products.

Given the global regulatory environment surrounding data protections for patients, our registry team will work with legal specialists to ensure patient data is appropriately protected according to international standards. For example, the EU General Data Protection Regulation will require additional protections for EU patients treated in the USA [42]. Healthcare organizations accepting patients from other countries will need to consider privacy and other protections associated with RWD.

The open dissemination of RWE to patients could raise their expectations of a possible treatment for their condition which cannot be met. Patients may also not realize the differences between possible scientifically-based regenerative medicine options from those clinics making false therapeutic promises with no scientific footing. To partially alleviate such concerns, we encourage medical and research organizations with open or published registry data to have a RMCS in order to properly educate and navigate patients to scientifically-based regenerative medicine options and help patients scrutinize different options offered at different institutions.

Finally, we recognize that the practice of regenerative medicine, and even ongoing clinical trials is still in its infancy and therefore, in order to collect meaningful outcomes numbers, data will need to be pooled by practitioners within a specific field and possibly even across medical specialties. Implementation of REBO and other similarly designed regenerative outcomes registries must have the capability to combine with each other in a manner that promote research while also protecting patient privacy. Many outcomes registry designers and vendors are currently addressing this very need.

\section{Conclusion}

Regenerative therapies are continuing to evolve at a rapid pace as medical researchers and providers innovate in a host of new scientific frontiers. Inherent in the practice of regenerative medicine is a shift away from how medical therapies are traditionally administered toward a more multidisciplinary approach. Several organizations have convened task forces or published position papers to promote the ethical use of cell and gene therapy. These include the International Society for Cell and Gene Therapy (ISCT), the International Society for Stem Cell Research (ISSCR) and the AABB's (formerly the American Association of Blood Banks) Center for Cellular Therapies whose goals include responsible innovation, professional development and patient safety [43-45]. Additionally, other clinically oriented professional groups such as the American Academy of Orthopedic Surgeons, the American Thoracic Society and the American Academy of Neurology have all published guidance statements to their clinician and scientist membership on the responsible translation of regenerative therapies [46-48].

Still to date, we see a need for more high-quality evidence (both in clinical trials and RWD) along with attention to quality control. Such data should include not only health outcomes, but also ethical and social science information in order to improve patient information and enhance ethical research. The optimal way to achieve these goals and the requisite responsible role out of regenerative therapies is still debated, but we propose one such framework for translation wherein the contributions of a diverse group of stakeholders with specific attention to scientific integrity, quality control and ethical concerns are to be considered. This includes enlisting multiple authorities with expertise in organ-specific disease conditions, cell therapy/cell biology, regulatory issues, ethical, legal and social aspects of regenerative medicine. Opportunities exist for the health research and healthcare fields to build and design the practice of regenerative medicine in a manner that adequately meets these goals.

\section{Acknowledgments}

The authors would like to thank the anonymous reviewers for helpful feedback on the manuscript.

\section{Financial \& competing interests disclosure}

The authors are employees of the Mayo Clinic. Generous support for this project was provided by the Mayo Clinic Center for Regenerative Medicine and the Louis V Gerstner Jr Fund at Vanguard Charitable. This publication was made possible by the Mayo 
Clinic CTSA through grant number: UL1 TR002377 from the National Center for Advancing Translational Sciences (NCATS), a component of the $\mathrm{NIH}$. The authors have no other relevant affiliations or financial involvement with any organization or entity with a financial interest in or financial conflict with the subject matter or materials discussed in the manuscript apart from those disclosed.

No writing assistance was utilized in the production of this manuscript.

\section{Open access}

This work is licensed under the Attribution-NonCommercial-NoDerivatives 4.0 Unported License. To view a copy of this license, visit http://creativecommons.org/licenses/by-nc-nd/4.0/

\section{References}

Papers of special note have been highlighted as: $\bullet$ of interest; $\bullet \bullet$ of considerable interest

1. Nelson TJ, Behfar A, Terzic A. Strategies for therapeutic repair: The " ${ }^{3}$ " regenerative medicine paradigm. Clin. Trans. Sci. 1(2), 168-171 (2008).

2. Terzic A, Nelson TJ. Regenerative medicine: advancing health care 2020. J. Am. Coll. Cardiol. 55(20), 2254-2257 (2010).

3. Terzic A, Nelson TJ. Regenerative medicine primer. Mayo Clin. Proc. 88(7), 766-775 (2013).

-• Highlights Mayo Clinic's vision for the development of regenerative therapies.

4. National Academies of Sciences, Engineering, and Medicine. Exploring the state of the science in the field of regenerative medicine: challenges of and opportunities for cellular therapies: proceedings of a workshop. The National Academies Press, DC, USA (2017). https://doi.org/10.17226/24671

5. Johnson S, Atala A. Regeneology: time for a new specialty? Stem Cell Transl. Med. 8(1), 4-6 (2019). http://dx.doi.org/10.1002/sctm.18-0101

-. Considers regenerative medicine as a new specialty.

6. Trounson A, DeWitt ND, Feigal EG. The alpha stem cell clinic: a model for evaluating and delivering stem cell-based therapies. Stem Cells Transl. Med. 1(1), 9-14 (2012).

- Describes the California Institute of Regenerative Medicine's Alpha Stem Cell Clinic model.

7. California Institute for Regenerative Medicine (CIRM). About alpha clinics (2018). www.cirm.ca.gov/patients/alpha-clinics-network/about

8. Kleiderman E, Boily A, Hasilo C, Knoppers BM. Overcoming barriers to facilitate the regulation of multi-centre regenerative medicine clinical trials. Stem Cell Res. Ther. 9, 307-316 (2018).

-. Outlines barriers to performing large scale, multi-center clinical trials.

9. US FDA. Statement from FDA Commissioner Scott Gottlieb, MD on FDA's comprehensive new policy approach to facilitating the development of innovative regenerative medicine products to improve human health (2017). www.fda.gov/newsevents/newsroom/pressannouncements/ucm585342.htm

10. US FDA. $21^{\text {st }}$ Century Cures Act (2018). www.fda.gov/regulatoryinformation/lawsenforcedbyfda/significantamendmentstothefdcact/21stcenturycuresact/default.htm

11. Surani SH, Levine AD. State performance in pluripotent and adult stem cell research, 2009-2016. Regen. Med. 13(3), 309-320 (2018).

12. Ronfard V, Vertès AA, May MH, Dupraz A, van Dyke ME, Bayon Y. Evaluating the past, present and future of regenerative medicine: a global view. Tissue Eng. Part B Rev. 23(2), 199-209 (2017).

- Updated evaluation of regenerative medicine therapies.

13. Siprashvili Z, Nguyen NT, Gorell ES et al. Safety and wound outcomes following genetically corrected autologous epidermal grafts in patients with recessive dystrophic epidermolysis bullosa. JAMA 1316(17), 1808-1817 (2016).

14. Hirsch T, Rothoeft T, Teig N et al. Regeneration of the entire human epidermis using transgenic stem cells. Nature 551, 327-332 (2017).

15. Holmes Iv JH, Molnar JA, Carter JE et al. A comparative study of the ReCell ${ }^{\circledR}$ device and autologous spit-thickness meshed skin graft in the treatment of acute burn injuries. J. Burn Care Res. 39(5), 694-702 (2018).

16. US FDA. FDA approval brings first gene therapy to the United States (2017). www.fda.gov/newsevents/newsroom/pressannouncements/ucm574058.htm

17. US FDA. FDA approves CAR-T cell therapy to treat adults with certain types of large B-cell lymphoma (2017). www.fda.gov/newsevents/newsroom/pressannouncements/ucm581216.htm

18. Jain MD, Davila ML. Concise review: emerging principles from the clinical application of chimeric antigen receptor $T$ cell therapies for B cell malignancies. Stem Cells 36(1), 36-44 (2018).

19. European Medicines Agency. First stem-cell therapy recommended for approval in EU (2014). www.ema.europa.eu/en/news/first-stem-cell-therapy-recommended-approval-eu 
20. Daniel E. EU approves first allogenic therapy for Crohn's disease. Pharmaceutical Technology (2018). www.pharmaceutical-technology.com/news/eu-allogenic-crohns-alofisel/

21. Houdek MT. Do early results of proximal humeral allograft-prosthetic composite reconstructions persist at 5-year followup? Clin. Orthop. Relat. Res. doi:10.1097/CORR.0000000000000417 (2018) (Epub ahead of print).

22. Dai WL, Zhou AG, Zhang H, Zhang J. Efficacy of platelet-rich plasma in the treatment of knee osteoarthritis: a meta-analysis of randomized controlled trials. Arthroscopy 33(3), 659-670 (2017).

23. Gentile P, Garcovich S, Bielli A, Scioli MG, Orlandi A, Cervelli V. The effect of platelet-rich plasma in hair regrowth: a randomized placebo-controlled trial. Stem Cell Transl. Med. 4(11), 1317-1323 (2015).

24 A futile cycle in cell therapy. Nat. Biotechnol. 35(4), 291 (2017).

25. Abbot S, Agbanyo F, Ahlfors JE et al. Report of the international conference on manufacturing and testing of pluripotent stem cells. Biologicals 56, 67-83 (2018).

26. Kamenova K, Caulfield T. Stem cell hype: media portrayal of therapy translation. Sci. Transl. Med. 7(278), 278ps4 (2015).

27. Sipp D, Caulfield T, Kaye J et al. Marketing of unproven stem cell-based interventions: a call to action. Sci. Transl. Med. 9(397), pii: eaag0426 (2017).

28. Turner L. The US direct-to-consumer marketplace for autologous stem cell interventions. Perspect. Biol. Med. 61(1), 7-24 (2018).

29. Knoepfler PS. The stem cell hard sell: report from a clinic's patient recruitment seminar. Stem Cell Transl. Med. 6(1), 14-16 (2017).

30. Scott CT. The case for stem cell counselors. Stem Cell Rep. 4(1), 1-6 (2015).

31. Master Z, Robertson K, Frederick D, Rachul C, Caulfield T. Stem cell tourism and public education: the missing elements. Cell Stem Cell 15(3), 267-270 (2014).

32. US FDA. Same surgical procedure exception under 21 CFR 1271.15(b): questions and answers regarding the scope of the exception, guidance for industry (2017).

www.fda.gov/downloads/biologicsbloodvaccines/guidancecomplianceregulatoryinformation/guidances/tissue/ucm419926.pdf

33. Shapiro SA, Kazmerchak SE, Heckman MG, Zubair AC, O'Connor MI. A prospective, single-blind, placebo-controlled trial of bone marrow aspirate concentrate for knee osteoarthritis. Am. J. Sports Med. 45(1), 82-90 (2017).

34. Shapiro SA, Arthurs JR, Heckman MG et al. Quantitative T2 MRI mapping and 12-month follow-up in a randomized, blinded, placebo controlled trial of bone marrow aspiration and concentration for osteoarthritis of the knees. Cartilagedoi:10.1177/1947603518796142 (2018) (Epub ahead of print).

35. Hay M, Thomas DW, Craighead JL, Economides C, Rosenthal J. Clinical development success rates for investigational drugs. Nat. Biotechnol. 32, 40-45 (2014).

36. Thomas DW, Burns J, Audette J, Carroll A, Dow-Hygelund C, Hay M. Clinical development success rates 2006-2015. BIO Industry Analysis. (2016). www.bio.org/sites/default/files/Clinical\%20Development\%20Success\%20Rates\%202006-2015\%20-\%20BIO,\%20B iomedtracker, \%20Amplion\%202016.pdf

37. Downing NS, Shah ND, Aminawung JA et al. Postmarket safety events among novel therapeutics approved by the US Food and Drug Administration between 2001 and 2010. JAMA 317(18), 1854-1863 (2017).

38. US FDA. CBER Regenerative Medicine Advanced Therapy (RMAT) designation requests (2018). www.fda.gov/BiologicsBloodVaccines/CellularGeneTherapyProducts/ucm587021.htm

39. US FDA. Use of real-world evidence to support regulatory decision-making for medical devices, Guidance for Industry and Food and Drug Administration Staff. (2017). www.fda.gov/downloads/medicaldevices/deviceregulationandguidance/guidancedocuments/ucm513027.pdf

- Discussees US FDA's regulatory perspectives on the use of real-world evidence for regulatory decision making.

40. Bipartisan Policy Center. Using real-world evidence to accelerate safe and effective cures: advancing medical innovation for a healthier America (2016). http://cdn.bipartisanpolicy.org/wp-content/uploads/2016/06/BPC-Health-Innovation-Safe-Effective-Cures.pdf

41. Registries for Evaluating Patient Outcomes: A User's Guide (Third Edition). Gliklich R, Dreyer N, Leavy M (Eds). Agency for Healthcare Research and Quality, Rockville, MD, USA (2014). www.effectivehealthcare.ahrq.gov/sites/default/files/related_files/registries-guide-3rd-edition-vol-2-140430.pdf

42. Davis J. Europe's GDPR privacy law is coming: here's what US health orgs need to know. Healthcare IT News. (2018). www.healthcareitnews.com/news/europes-gdpr-privacy-law-coming-heres-what-us-health-orgs-need-know

43. International Society for Cell and Gene Therapy (ISCT). The ISCT Presidential Task Force (PTF) on the use of unproven and/or unethical cell and gene therapy (2018). www.isct-unprovencellulartherapies.org

44. ISSCR Guidelines for stem cell research and clinical translation (2016). www.isscr.org/docs/default-source/all-isscr-guidelines/guideline s-2016/isscr-guidelines-for-stem-cell-research-and-clinical-translationd67119731dff6ddbb37cff0000940c19.pdf?sfvrsn=4

- Internationally recognized guidelines for the responsible translation of stem cell-based interventions.

45. AABB. Center for cellular therapies (2018). www.aabb.org/aabbcct/Pages/default.aspx 
46. Chu CR, Rodeo S, Bhutani N et al. Optimizing clinical use of biologics in orthopedic surgery: consensus recommendations from the 2018 AAOS/NIH U-13 Conference. J. Am. Acad. Orthop. Surg. 27(2), e50-e63 (2019).

47. American Thoracic Society. Unproven stem cell treatments for lung disease - an emerging public health problem. Am. J. Respir. Crit. Care Med. 195, 13-14 (2017).

48. Mihalko WM, Golish SR, Watson JT. Stem cell centers in the United States: patients should proceed with caution. American Academy of Orthopedic Surgeons (2018). www.aaos.org/News/DailyEdition2018/Friday/004/?ssopc=1 